\title{
METAS SOCIALES EN LAS CLASES DE EDUCACIÓN FÍSICA
}

Juan Antonio Moreno Murcla*

Davld González-Cutre Coll**

Álvaro Sicilia Camacho**

Facultad de Cienclas del Deporte, Unlversidad de Murcia*

Facultad de Humanidades y Ciencla de la Educación, Universidad de Almería"

\section{RESUMEN}

Investigaciones recientes señalan que las metas sociales deben ser tenidas en cuenta en el estudio de la motivación (e.g. Anderman y Anderman, 1999; Urdan y Maehr, 1995). El objetivo de este trabajo fue validar al contexto español la única escala disponible para la evaluaclón de las metas sociales en clases de educación fisica: la Social Goal Scale-Physical Education (Guan, McBride, y Xiang, 2006), a través de dos estudios con muestras de 406 y 813 alumnos de educación física respectivamente. Se analizaron las propiedades psicométricas de la escala realizando análisis de la consistencia interna mediante el alfa de Cronbach, análisis factoriales confirmatorios y test de invarianza por género. Finalmente se buscaron evidencias externas de validez utilizando la variable "competencia percibida". El análisis de los datos mostro que la escala era válida y fiable para su utilización con adolescentes españoles en clases de educación fisica. Además, los resultados revelaron que la meta social de responsabilidad predecla positiva y significativamente la competencia percibida.

Correspondencia: Juan Antonio Moreno Murcla. Facultad de Clencias del Deporte. Universidad de Murcia. Parque Almansa. 30730 San Javiar, Murcia, Espafía. E-mail: morenomu@um.es. Tel: 968398678 . Fax: 968398672 
Palabras clave: METAS SOCIALES, META DE RELACIÓN, META DE
RESPONSABILIDAD, EDUCACIÓN FÍSICA, COMPETENCIA PERCIBIDA.

\section{SUMMARY}

Recent investigations point out that social goals should be taken into account in the study of motivation (e.g. Anderman \& Anderman, 1999; Urdan \& Maehr, 1995). The objective of this paper has been to validate in the Spanish context the only available scale for the evaluation of social goals in physical education classes: the Social Goal Scale-Physical Education (Guan, McBride, \& Xiang, 2006). To achieve this, two studies with samples of 406 and 813 students in physical education classes were carried out. The psychometric properties of the scale were analyzed by carrying out some checks of intemal consistency through the Cronbach alpha, some confirmatory factor analyses and a test of invariance across gender. Finally, external evidences of validity were looked for by using the "perceived competence variable. The analysis of the data showed that the scale was valid and reliable for its use with Spanish adolescents in physical education classes. Also, the results revealed that social responsibility goal positively and significantly predicted perceived competence.

\section{Koy words: SOCIAL GOALS, RELATIONSHP GOAL, RESPONSBBITYGOAL, PHYSICAL EDUCATON, PERCENED COMPETENCE}

\section{INTRODUCCIÓN}

Durante las últimas dos décadas los investigadores en el ámbito de la psicologia de la actividad fisica y del deporte han utilizado la teoria de las metas de logro (Nicholls, 1989) como marco de referencia para analizar la motivación y la búsqueda de éxito en las clases de educación física (Carpenter y Morgan, 1999; Chen, 2001; Papaioannou, 1998; Spray y Biddle, 1997). Según esta teorla las personas pueden definir el éxito como superación y mejora personal, esfuerzo e implicación (orientación de metas a la tarea), o bien como superación de los demás 
y consecución de mejores resultados (orientación de metas al ego), siendo ambas compatibles. Pero el análisis de la motivación de logro no se puede limitar a esta teoria, ya que diferentes trabajos realizados en ámbitos académicos (e.g. Anderman y Anderman, 1999; Hicks, 1996; Urdan y Maehr, 1995; Wentzel, 1991) señalan que los estudiantes pueden tener razones sociales que determinen su éxito. Por ejemplo, un alumno puede considerar que tiene éxito si consigue ser una persona responsable que se comporta acorde a unos valores establecidos por la sociedad, o si logra tener un grupo de amigos con el que pasarto bien y compartir cosas. Especialmente en la adolescencia, no se puede analizar el compromiso acadérnico sin tener en cuenta las metas sociales, ya que en esta etapa se incrementarı las preocupaciones sociales de los alumnos y cobra gran importancia la relación con el grupo de iguales (Urdan y Maehr, 1995).

La perspectiva ecológica consideró el aula como un nicho compuesto por diferentes sistemas que interactúan entre sl, siendo uno de ellos el sistema de interacciones sociales (Doyle, 1983; Siedentop, 1998). Junto con los sistemas instruccional y organizativo, el trabajo de Tinning y Siedentop (1985) refiejó la importancia que para la educación física tenía el sistema de interacción social, dado que suele ser iniciado y mantenido por el alumnado y generalmente utilizado por ellos como instrumento de negociación en el aula. En un trabajo más reciente Carlson (1995) y Carlson y Hastie (1997) destacaron la importancia del sistema social en educación física y las múltiples relaciones que encuentra con la estructura de tareas. Concretamente, al analizar desde el punto de vista del estudiante el aula de educación física los autores pudieron apreciar que el alumnado se comportaba de acuerdo a la consecución de dos grandes objetivos: pasárselo bien y superar el curso. Mientras el primer objetivo dependía en mayor grado de las relaciones con los compañeros, la superación del curso estaba más asociada a cumplir las exigencias establecidas por el profesor en torno a la estructura de tareas. Desde el campo de la psicología educativa han existido en la última década intentos por integrar las metas sociales al estudio de la motivación, dado que esta dimensión estaría afectando al comportamiento y rendimiento de los estudiantes (Anderman y Anderman, 1999; Hicks, 1996; Guan, McBride, y Xiang, 2006; Patrick, Hicks, y Ryan, 1997). 
Las metas sociales se pueden definir como representaciones cognitivas de resultados propuestos y deseados en el dominio social (Hicks, 1996). Los trabajos realizados en ámbitos educativos (Anderman y Anderman, 1999; Guan, McBride, y Xiang, 2006; Patrick y cols., 1997; Ryan, Hicks, y Midgley, 1997) establecen la existencia de al menos tres metas sociales: la meta de responsabilidad, la meta de relación y la meta de estatus. La meta de responsabilidad refleja el deseo de respetar las reglas sociales y el rol establecido (Wentzel, 1991), la meta de relación hace referencia al deseo de mantener buenas relaciones con los compañeros, y la meta de estatus se centra en la búsqueda de relación con el grupo de gente más popular de la clase.

La meta social de responsabilidad puede estar asociada con patrones motivacionales adaptativos. De hecho, en el estudio longitudinal con 660 estudiantes, llevado a cabo por Anderman y Anderman (1999), se evidencia que los sujetos con metas sociales de responsabilidad dan más importancia al aprendizaje, a la mejora personal y al esfuerzo; existiendo una clara relación entre dicha meta y la orientación a la tarea. Por el contrario, aquellos estudiantes que reflejaron metas de relación y estatus, estuvieron más orientados al ego y, al mismo tiempo, trataron de demostrar mayor habilidad que los demás. Otras investigaciones han demostrado, sin embargo, que los alumnos con metas de responsabilidad alcanzan mayores calificaciones en la escuela y se perciben con mayor auto-eficacia (Patrick y cols., 1997; Wentzel, 1993). En este sentido, Urdan y Maehr (1995) afirman que las relaciones entre las diferentes metas sociales y las percepciones de competencia o auto-eficacia del alumno, deben ser uno de los aspectos a analizar en futuras investigaciones.

En contextos de educación fisica algunos estudios han destacado también la importancia de desarrollar metas sociales de responsabilidad, al objeto de conseguir consecuencias más positivas para el aprendizaje. Asi, recientemente Guan, Xiang, McBride, y Bruene (2006) han encontrado una asociación positiva y significativa de las metas de relación y responsabilidad con diferentes metas de logro. Este último estudio también ha revelado que la meta de responsabilidad predice positiva y significativamente el esfuerzo y la persistencia manifestada por ios estudiantes. 
Existen hasta la fecha diferentes intentos por crear instrumentos que puedan evaluar las metas sociales en el ámbito flsico-deportivo, si bien la definición del constructo todavla es problemática, encontrándose en los trabajos diferentes metas que podrían estar solapándose. Partiendo de una extensa revisión de la bibliografía especializada, Allen (2003) elaboró una escala para medir las metas sociales en relación al deporte con jóvenes. Dicha escala fue denominada Escala de las Orientaciones Motivacionales Sociales para el Deporte (Social Motivational Orientations Scale for Sport, SMOSS) y se compone de 15 items agrupados en tres factores: «orientación a la afiliación" (haciendo referencia al desarrollo de relaciones sociales Intimas y a la diversión con otros, e.g. «hago algunos buenos amigos en el equipo", «me divierto con los otros en mi equipo"), uestatus social» (reflejando la búsqueda de popularidad entre los compañeros, e.g. «pertenezco al grupo popular del equipo»); y kreconocimiento social» (enfatizando el reconocimiento de los demás, e.g. «otros me dicen que lo he hecho bieny). El primer factor se compone de siete Items, mientras que los otros dos se componen de cuatro items cada uno. La escala va encabezada por el enunciado «Siento que las cosas han ido bien en mi deporte cuando.... y, y se responde con una escala tipo Likert de 1 (totalmente en desacuerdo) a 5 (totalmente de acuerdo).

Del mismo modo, Stuntz y Weiss (2003) desarrollaron 15 Items para medir las metas sociales con deportistas adolescentes. Dichos ítems están encabezados por la sentencia «Siento que el deporte me ha ido realmente bien cuando...», y se agrupan en tres factores: amistad (e.g. ktengo una amigo intimo en el deporte que se preocupa por mis sentimientos"), aceptación de los compañeros (e.g. «Todos en el equipo me quieren como amigow) y elogio del entrenador (e.g. «Mi entrenador elogia mi actuación»), respondiéndose mediante una escala tipo Likert de 1 a 5.

Recientemente, Guan, McBride, y Xiang (2006) han elaborado una escala para analizar las metas sociales en el contexto de la educación fisica. Para este fin, los autores adaptaron la escala de metas sociales (Social Goal Scale, SGS) utilizada por Patrick y cols. (1997) en el ámbito académico. Dicha escala, denominada Social Goal ScalePhysical Education (SGS-PE), está compuesta por un total de 11 items, encabezados por la frase «En mis clases de educación fisica», y 
agrupados en dos factores: meta de relación (seis items) y meta de responsabilidad (cinco items). Los diferentes items se responden mediante una escala tipo Likert de 1 (totalmente en desacuerdo) a 7 (totalmente de acuerdo). El análisis de sus propiedades psicométricas a través de dos estudios reveló coeficientes alfa de .76 y .73 para la meta de relación y .79 y .84 para la meta de responsabilidad. En el primer estudio se realizó un análisis factorial exploratorio obteniendo un porcentaje de varianza explicada del $54.70 \%$ y en el segundo estudio un análisis factorial confirmatorio en el que se obtuvieron Indices aceptables: $\chi^{2} / g . l$. $=3.55$; CFI (Comparative Fit Index) $=.93 ; \mathrm{GFI}$ (Goodness of Fit Index) = .94; TLI (Tucker Lewis Index) = .90; RMSEA (Root Mean Square Error of Aproximation) = .08. Además, se demostró la invarianza del modelo entre las dos muestras de estudlo.

Diferentes trabajos han mostrado que las metas sociales deben ser un elemento a tener en cuenta en el estudio de la motivación (e.g. Urdan y Maehr, 1995). No obstante, hasta la fecha la única escala validada para evaluar las metas sociales en educación fisica es la SGS-PE de Guan, McBride, y Xiang (2006), habiendo reflejado unas adecuadas propiedades psicométricas. Con estos antecedentes, el principal objetivo de este trabajo es validar la SGS-PE al contexto español con estudiantes adolescentes, lo que permitiría en un futuro analizar en nuestro pals el constructo de metas sociales en las clases de educación fisica.

\section{ESTUDIO 1: DESARROLLO PSICOMÉTRICO DE LA SGS-PE EN EL CONTEXTO ESPANOL}

El objetivo de este primer estudio fue adaptar al contexto español la Social Goal Scale-Physical Education. Se trató de testar su estructura factorial a través de un análisls factorial confirmatorio y se examinó su consistencia interna.

\section{MÉTODO}

\section{Muestra}

En este estudio participaron un total de 406 alumnos en clases de educación física con edades entre 11 y 16 años $(M=12.64, D T=1.04)$, 
de los cuales 220 eran chicos y 186 chicas, de diferentes centros de enseñanza localizados en la ciudad de Murcia. Cabe destacar que tanto los centros de enseñanza a los que se acudio, como el profesorado y alumnado participaron voluntariamente en el desarrollo de la investigación.

\section{Instrumentos}

Escala de Metas Sociales en Educación Fisica (EMSEF). Se empleó la traducción al castellano (ver Apéndice) de la Social Goal Scale-Physical Education (SGS-PE) de Guan, McBride, y Xiang (2006). Esta escala estaba compuesta por un total de 11 ftems agrupados en dos factores, meta de relación (seis items) (e.g. «me gustaría llegar a conocer muy bien a mis amigos/as de clasen) y meta de responsabilidad (cinco Items) (e.g. «intento hacer lo que el profesor/a me pide que haga»), que se respondlan mediante una escala tipo Likert de 1 (totalmente en desacuerdo) a 7 (totalmente de acuerdo).

\section{Procedimiento}

Adoptamos una estrategia de traducción inversa (Hambleton, 1996), de tal forma que los items de la SGS-PE fueron traducidos al español, para que posteriormente un traductor ajeno al grupo de investigación los volviera a traducir al inglés. Se compararon los Items traducidos al inglés con los de la escala original, observando en dicha comparación la total coincidencia. Seguidamente, los diferentes items fueron evaluados por tres expertos en la materia (Lynn, 1986), que conslderaron que eran pertinentes para medir las metas sociales de relación y responsabilidad, y que la redacción era correcta.

A continuación, nos pusimos en contacto con el equipo directivo de los centros de enseflanza escogidos, para pedirles su colaboración en este estudio. Se solicitó a los alumnos una autorización por escrito de sus padres para participar en el estudio, debido a su minoria de edad. De forma previa a la investigación, la escala fue administrada a un reducido grupo de estudiantes para verificar su correcta comprensión, y en caso contrario llevar a cabo los cambios pertinentes.

La administración de la escala definitiva se realizó en presencia del investigador principal, para explicar de forma breve su objetivo y 
estructura, así como la forma de rellenarla. Durante el proceso de cumplimentación el investigador principal solventó todos aquellos problemas que pudieron surgir. En este estudio se respetaron todos los procedimientos éticos para la recogida de datos.

\section{Análisis de datos}

Se llevó a cabo un análisis inicial de las propiedades psicométricas de la escala. En primer lugar se realizó un análisis de la consistencia interna a través del alfa de Cronbach y seguidamente un análisis factorial confirmatorio para comprobar si la estructura de dos factores (relación y responsabilidad), con sus correspondientes ítems, se ajustaba correctamente al contexto español.

Resultados

Análisis de la consistencia interna. Los resultados mostraron un coeficiente alfa de .75 para el factor relación y .73 para el factor responsabilidad.

Análisis Factorial Confirmatorio. Para testar el modelo de metas sociales efectuamos un análisis factorial confirmatorio (AFC) (Figura 1). Hay poco acuerdo entre los investigadores para determinar cuál es el mejor índice de ajuste a utilizar en el AFC (Hoyle y Panter, 1995), por eso, lo más adecuado para aceptar o rechazar un modelo es emplear una combinación de varios Indices. Así pues, tuvimos en cuenta algunos de los indices de ajuste más utilizados: $\chi^{2}, \chi^{2} / g . l$, CFI (Comparative Fit Index), NFI (Normed Fit Index), TLI (Tucker Lewis Index), RMSEA (Root Mean Square Error of Aproximation) y SRMR (Standardized Root Mean Square Residual). El $\chi^{2}$ evalúa el ajuste absoluto del modelo a la matriz de covarianza, pero es muy sensible al tamaño muestral (Jöreskog y Söbom, 1989). Para controlar esto se emplé también el $\chi^{2} / g . l$., que se considera aceptable cuando es inferior a 5 (Bentler, 1989). Los índices incrementales (CFI, NFI y TLI) se basan en la comparación entre el modelo hipotetizado y el modelo nulo, y no se ven afectados por el tamaño de la muestra, considerándose aceptables valores superiores a .85, aunque lo ideal son valores superiores a .90 (Ntoumanis, 2001). EI RMSEA y SRMR son los indices de error y deben ser inferiores a .07 para considerarlos aceptables (Ntoumanis, 2001). Los índices obtenidos en este estudio 
indican un ajuste adecuado para la escala: $\chi^{2}(43, N=406)=121.59$, $p=.00 ; \chi^{2} / g . l .=2.82 ; \mathrm{CFI}=.93 ; \mathrm{NFI}=.89 ; \mathrm{TLI}=.91 ; \mathrm{RMSEA}=.06$; $\mathrm{SRMR}=.04$.

Figura 1. Análisis factorial confirmatorio de la EMSEF. Las elipses representan los factores, y los rectángulos representan los diferentes Items. Todos los parámetros están estandarizados y son significativos $(p<.05)$. Las varianzas residuales se muestran en pequeños círculos

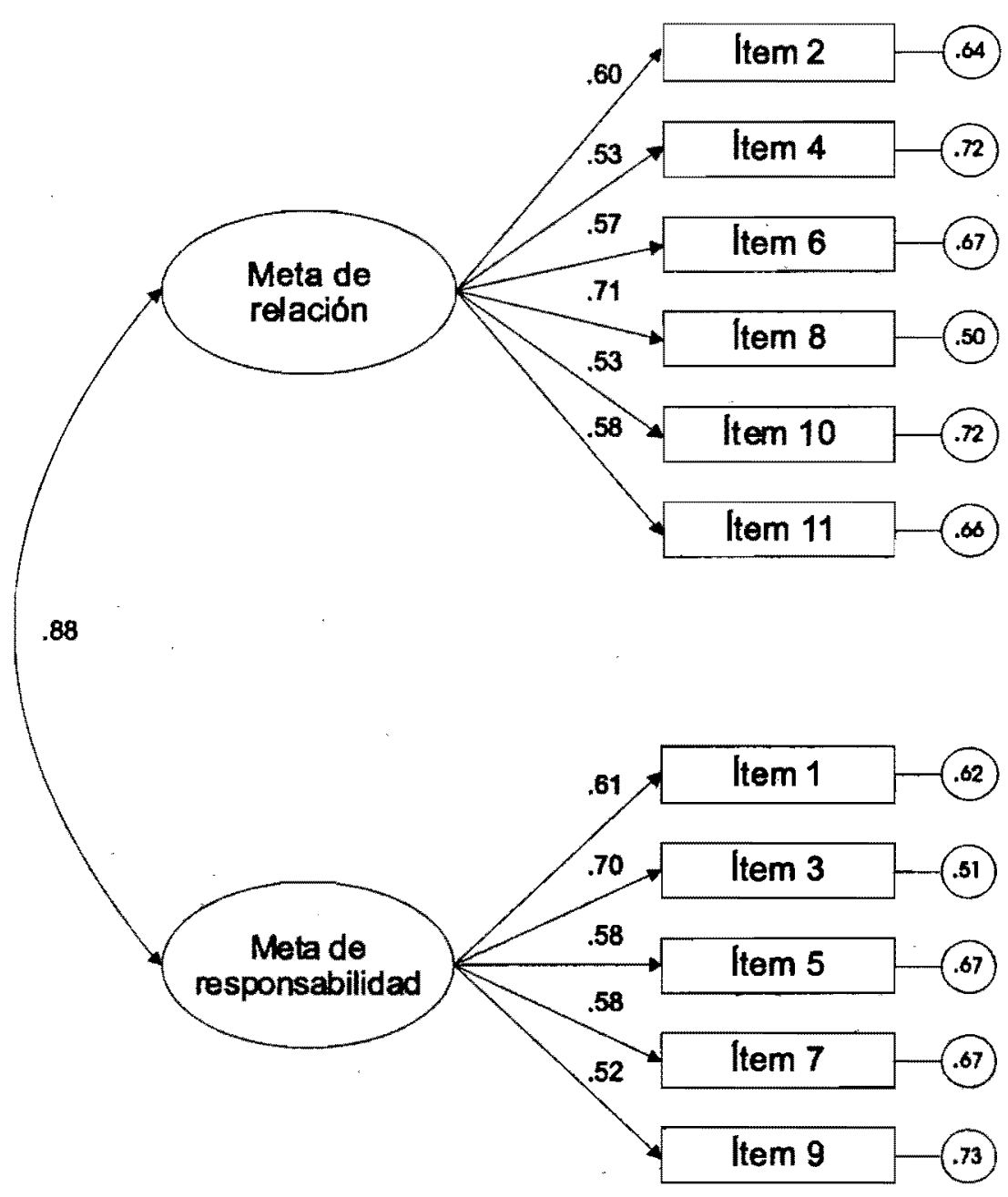




\section{ESTUDIO 2: TESTS PSICOMÉTRICOS ADICIONALES Y PREDICCION DE LA COMPETENCIA PERCIBIDA}

En este segundo estudio se pretendió confirmar de nuevo la estructura factorial de la escala y analizar su consistencia interna utilizando una muestra más amplia. Además se trató de comprobar la invarianza del modelo por género y se examinó la validez externa relacionando las metas sociales con la competencia percibida.

\section{MÉTODO}

\section{Muestra}

Estuvo compuesta por 813 alumnos (420 chicos y 393 chicas), pertenecientes a un total de 16 centros situados en la ciudad de Murcia. Las edades estuvieron comprendidas entre los 11 y 16 años $(M=$ 12.93, DT = 1.02).

\section{Proced Imiento e instrumentos}

La administración de los cuestionarios se ajustó a las consideraciones contempladas en el Estudio 1. Los instrumentos utilizados fueron los siguientes:

Escala de Metas Sociales en Educación Fisica (EMSEF). Se utilizó la versión en castellano de la SGS-PE (Guan, McBride, y Xiang, 2006), denivada del modelo del Estudio 1.

Competencia percibida. Para obtener evidencias externas de validez de la EMSEF, se utilizó el factor «competencia deportiva» de la versión española (Moreno y Cervello, 2005) del Physical SelfPerception Profile (Fox, 1990; Fox y Corbin, 1989). Dicho factor se componla de seis ítems que se respondian mediante una escala tipo Likert de 1 (totalmente en desacuerdo) a 4 (totalmente de acuerdo). En este estudio el factor "competencla percibida" reveló un coeficiente alfa de .86 y los siguientes indices en el análisis factorial confirmatorio: $\chi^{2}(8, N=813)=52.45, p=.00 ; \chi^{2} / g . l .=6.55$; $\mathrm{CFI}=.97 ; \mathrm{NFI}=.97 ; \mathrm{TLI}=.95 ; \mathrm{RMSEA}=.08 ; \mathrm{SRMR}=.02$. El valor del $\chi^{2} / g . l$. superior a 5 se puede justificar por el gran tamaño de la 
muestra, ya que a pesar del ajuste por grados de libertad, perdura el efecto negativo del tamaño muestral en el índice de ajuste (Cea, 2002). Respecto al RMSEA, algunos autores consideran que se pueden aceptar valores inferiores a .10 (Browne y Cudeck, 1993), por lo que el modelo parece adecuado.

\section{Anállsis de datos}

Inicialmente se analizó la fiabilidad de la escala mediante el alfa de Cronbach y se realizó un análisis factorial confirmatorio. A continuación se llevó a cabo un test de invarianza por género. Finalmente se buscaron evidencias de validez externa de la EMSEF, tratando de analizar el poder de predicción de las metas sociales de relación y responsabilidad sobre la competencia percibida a través de un modelo de ecuaciones estructurales. Se eligio la variable competencia percibida para analizar su relación con las metas sociales siguiendo las recomendaciones de Urdan y Maehr (1995) sobre las variables a tener en cuenta en el análisis de las metas sociales, $y$ apoyándonos en algún estudio previo como el de Patrick y cols. (1997).

\section{Resultados}

Estimación de la fiabilidad. Los resultados mostraron valores similares al Estudio 1, con un coeficiente alfa de .75 para el factor relación y .72 para el factor responsabilidad.

Análisis factorial confirmatorio y test de invarianza por género. El análisis factorial confirmatorio se realizó siguiendo el mismo modelo que en el Estudio 1 y atendiendo a los mismos Indices de ajuste. Partiendo de los valores de corte descritos previamente, los Indices obtenidos en este estudio fueron aceptables y similares a los obtenidos en el Estudio $1: \chi^{2}(43, N=813)=198.85, p=.00 ; \chi^{2} / g . \mathrm{l}=4.62 ; \mathrm{CFI}=$ $.92 ; \mathrm{NFI}=.91 ; \mathrm{TLI}=.90 ; \mathrm{RMSEA}=.06 ; \mathrm{SRMR}=.04$. Los índices de ajuste del modelo para los chicos y las chicas también se mostraron aceptables (Tabla 1). La Tabla 2 presenta los estadisticos de ajuste para el test de invarianza simultáneo por género. Estos resultados proporcionan un fuerte apoyo a favor de la invarianza del modelo de medida de la EMSEF por género. 
Evidencias externas de validez: predicción de la competencia percibida. El análisis a través del modelo de ecuaciones estructurales señalo que la meta de responsabilidad predecia la competencia percibida de forma positiva y significativa $(=.43)$, mientras que la meta de relación no lo hacla de forma significativa. Los indices obtenidos fueron: $\chi^{2}(117, N=813)=396.46, p=.00 ; \chi^{2} / g . l .=3.38$; $\mathrm{CFI}=.93 ; \mathrm{NFI}=.90 ; \mathrm{TLI}=.92 ; \mathrm{RMSEA}=.05 ; \mathrm{SRMR}=.04$, lo que indica que los datos se ajustan al modelo propuesto.

Tabla 1. Indices de ajuste del análisis factorial confirmatorio de la EMSEF porgénero

\begin{tabular}{lcc}
\hline & Chicos & Chicas \\
\hline$n$ & 420 & 393 \\
$\chi^{2}(43)$ & 108.34 & 119.63 \\
$\chi^{2} / g . l$. & 2.52 & 2.78 \\
CFI & .94 & .92 \\
NFI & .91 & .88 \\
TLI & .93 & .90 \\
RMSEA & .06 & .06 \\
SRMR & .04 & .05 \\
\hline
\end{tabular}

Tabla 2. Indices de ajuste para el análisis multi-grupo de la EMSEF

\begin{tabular}{lc}
\hline & Género \\
\hline$\chi^{2}$ & 136.54 \\
Grados de libertad & 52 \\
$\chi^{2} / g . l$. & 2.62 \\
CFI & .94 \\
NFI & .90 \\
TLI & .91 \\
RMSEA & .07 \\
SRMR & .04 \\
\hline
\end{tabular}




\section{DIScusión}

El propóslto de este estudio ha sido adaptar la SGS-PE al contexto español. Para testar sus propiedades psicométricas se realizaron dos estudios que incluyeron coeficientes alfa de Cronbach, AFC, test de invarianza por género y análisis de la validez externa a través de la varlable competencia percibida. Los dos factores que componen la escala (meta de relación y meta de responsabilidad) obtuvieron en arnbos estudios un alfa de Cronbach superior a .70, lo que nos indica que la escala posee una consistencia interna fiable para las dos muestras de estudiantes adolescentes españoles de educación fislca. Los resultados del AFC en los dos estudios realizados revelaron indices de ajuste dentro de un rango aceptable $y$, por tanto, parece ser que la escala proporciona puntuaciones validas. Los resultados obtenidos son similares a los de la escala orlginal (Guan, McBride, y Xiang, 2006) validada en Estados Unidos. Además, se observa que el modelo propuesto estuvo bien representado tanto en los chlcos como en las chlcas. Asl pues, el anállsis de las propledades psicométricas de la Escala de Metas Sociales en Educación Fisica (EMSEF) revela que dicha escala es apropiada para valorar las metas sociales con estudiantes adolescentes de educaclón fisica en el contexto español.

Para buscar evidencias externas de validez, seguidamente analizamos las relaciones existentes entre las metas sociales y la competencla perclbida. Los resultados Indicaron que la meta de responsabilidad predecla positiva y significativamente la competencia percibida mlentras que la meta de relactón no lo hacia. En línea con el trabajo de Patrick y co/s. (1997), nuestros resultados indican que los estudiantes adolescentes con metas de responsabilidad revelan una mayor auto-eficacia percibida para el trabajo académico que sus compañeros. Por otro lado, la meta de responsabilidad mantiene relación con algunas consecuencias positivas en el aula (esfuerzo, persistencia e interés por el aprendizaje), tal y como sucede con la competencia percibida (Anderman y Anderman, 1999; Guan, Xlang y cols., 2006). Estos resultados sugieren que los docentes deberlan tratar de desarroflar metas de responsabilidad en los estudiantes para consegulr efectos positivos en su implicación y aprendizaje. 
Aunque en este trabajo no se han encontrado relaciones sigrificativas entre la competencia percibida y la meta de relación, futuros estudios deberán continuar profundizando en el análisis de estas relaciones. Teniendo en cuenta que la competencia percibida es una dimensión del autoconcepto, y que en las clases de educación fisica las relaciones sociales suelen ser más valoradas y visibles que en otras situaciones de aula (Carlson, 1995; Tinning y Siedentop, 1985; Siedentop, 1998), es posible que el alumnado muestre menos inconveniente a exponerse a relaciones con los demás cuanto más competente se perciba respecto a las tareas del aula.

Dado las implicaciones que las metas sociales pueden tener en la motivación en contextos de educación física, es importante entender su papel para conseguir una mayor adherencia de los adolescentes a la práctica de actividades fisicas y deportivas. La aportación principal de este trabajo ha sido la de corroborar la validez y fiabilidad de la SGS-PE, lo que permitirá medir en España las metas sociales en estudiantes de educación física. Supone, además, una contribución importante al campo de la investigación en motivación deportiva, ya que va a permitir que futuros estudios analicen de forma conjunta cómo las diferentes metas sociales y metas de logro se relacionan con determinadas consecuencias afectivas, cognitivas y conductuales. No obstante, debemos reconocer que en este estudio sólo se han tenido en cuenta las metas sociales de responsabilidad y relación. Futuros trabajos deberian tratar de evaluar otras metas sociales (como la de estatus o reconocimiento social), clarificando algo más este complejo entramado teórico. En esta línea, sería interesante evaluar la relación de las metas sociales con algunos precedentes y consecuentes existentes en la bibliografla consultada. Así, por ejemplo, el estudio de la relación entre metas sociales y metas de logro podría dilucidar si el clima motivacional creado por los profesores de educación física afecta a determinadas metas sociales. En el mismo sentido, parece interesante determinar las relaciones entre las metas sociales y algunas de las variables que estarian afectando al aprendizaje académico en educación física, tales como la motivación autodeterminada, el esfuerzo, el interés, la persistencia, el flow o estado psicológico óptimo, o la adherencia a la práctica. También en futuras investigaciones sería interesante intentar adaptar esta escala al ámbito 
de las escuelas deportivas. Al tratarse de otro entorno educativo, podria determinarse si las metas sociales de los jóvenes deportistas influyen de manera importante en su compromiso deportivo. Finalmente, trabajos futuros deberian analizar la estabilidad de las metas sociales, intentando apreciar diferencias por género, edad, cultura, nivel de práctica deportiva, y otras posibles variables demográficas. En este sentido, los estudios podrian dilucidar si a una determinada edad las metas sociales se convierten en un rasgo disposicional dificil de modificar (Allen, 2003). Obviamente la validación de una escala es un proceso continuado y, por tanto, son necesarios más trabajos que analicen las propiedades psicométricas de la escala con diferentes muestras, culturas y análisis estadísticos, para asi determinar si puede ser una herramienta útil a la hora de medir las metas sociales en las clases de educación física.

\section{REFERENCIAS BIBLIOGRÁFICAS}

Allen, J. B. (2003). Social motivation in youth sport. Journal of Sport and Exercise Psychology, 25, 551-567.

Anderman, L. H., y Anderman, E. M. (1999). Social predictors of changes in students' achievement goal orientations. Contemporary Educational Psychology, 25, 21-37.

Bentler, P. M. (1989). EQS structural equations program manual. Los Angeles: BMDP Statistical Software.

Browne, M. W., y Cudeck, R. (1993). Alternative ways of assessing model fit. En K. A. Bollen, y J. S. Long (Eds.), Testing structural equation models (pp. 136-162). Newbury Park, California: Sage.

Carlson, T. B. (1995). We hate gym: Student alienation from physical education. Journal of Teaching in Physical Education, 14, 467-477.

Carlson, T. B., y Hastie, P. (1997). The student social system within sport education. Joumal of Teaching in Physical Education, 16, 176195.

Carpenter, P. J., y Morgan, K. (1999). Motivational climate, personal goal perspectives and cognitive and affective responses in physical education classes. European Joumal of Physical Education, 4, 31-44. Cea, M. A. (2002). Análisis multivariable. Teoria y práctica en la investigación social. Madrid: Sintesis. 
Chen, A. (2001). A theoretical conceptualization for motivation research in physical education: An integrated perspective. Quest, 53, 35-38. Doyle, W. (1983). Academic work. Review of Educational Research, $53,159-199$.

Fox, K. R. (1990). The Physical Self-Perception Profile manual. DeKalb: Office for Health Promotion, Northem Illinois University.

Fox, K. R., y Corbin, C. D. (1989). The Physical Self-Perception Profile: Development and preliminary validation. Journal of Sport and Exercise Psychology, 11, 408-430.

Guan, J., McBride, R. E., y Xiang, P. (2006). Reliability and validity evidence for the Social Goal Scale-Physical Educatlon (SGS-PE) in high school settings. Journal of Teaching in Physical Education, 25, 226-238.

Guan, J., Xiang, P., McBride, R., y Bruene, A. (2006). Achievement goals, social goals and students' reported persistence and effort in high school physical education. Joumal of Teaching in Physical Education, 25, 58-74.

Hambleton, R. K. (1996). Adaptación de tests para su uso en diferentes idiomas y culturas: fuentes de error, posibles soluciones y directrices prácticas. En J. Muñiz (Ed.), Psicometria (pp. 207-238). Madrid: Universitas.

Hicks, L. (1996). Social goals in early adolescence: Development, gender, and schooling as contexts. Unpublished doctoral dissertation, University of Michigan, USA.

Hoyle, R. H., y Panter, A. T. (1995). Writing about structural equation models. En R. H. Hoyle (Ed.), Structural equation modeling: concepts, issues, and applications (pp. 158-176). Thousand Oaks, CA: Sage.

Jöreskog, K. G, y Sörbom, D. (1989). LISREL 7: A guide to the program and applications $\left(2^{*}\right.$ ed.). Chicago: SPSS.

Lynn, M. (1986). Determination and quantification of content validity. Nursing Research, 35, 382-385.

Moreno, J. A., y Cervell6, E. (2005). Physical self-perception in Spanish adolescents: effects of gender and involvement in physical activity. Journal of Human Movement Studies, 48, 291-311.

Nicholls, J. G. (1989). The competitive ethos and democratic education. Cambridge, MASS: Harvard University Press. 
Ntoumanis, N. (2001). A self-determination approach to the understanding of motivation in physlcal education. British Journal of Educational Psychology, 71, 225-242.

Papaioannou, A. (1998). Students' perceptions of the physical education class environment for boys and girls and the perceived motivational climate. Research Quarterly for Exercise and Sport, 69, 267-275.

Patrick, H., Hicks, L., y Ryan, A. M. (1997). Relations of perceived social efficacy and social goal pursuit to self-efficacy for academic work. Joumal of Early Adolescence, 17, 109-128.

Ryan, A. M., Hicks, L., y Midgley, C. (1997). Social goals, academic goals, and avoiding seeking help in the classroom. Joumal of Early Adolescence, 17, 152-171.

Siedentop, D. (1998). Aprender a enseñar la educación física. Barcelona: Inde.

Spray, C. M., y Biddle, S. J. H. (1997). Achievement goal orientations and participation in physical education among male and female sixth form students. European Physical Education Revlew, 3, 8390.

Stuntz, C. P., y Weiss, M. R. (2003). Influence of social goal orientations and peers on unsportsmanlike play. Research Quarterly for Exercise. and Sport, 74, 421-435.

Tinning, R., y Siedentop, D. (1985). The characteristics of tasks and accountability in student teaching. Joumal of Teaching in Physical Education, 4, 286-299.

Urdan, T. C., y Maehr, M. L. (1995). Beyond a two-goal theory of motivation and achievement: A case for social goals. Review of Educational Research, 65, 213-243.

Wentzel, K. R. (1991). Social competence at school: Relation between social responsibility and academic achievement. Review of Educational Research, 61, 1-24.

Wentzel, K. R. (1993). Motivation and achievement in early adolescence: The role of multiple classroom goals. Joumal of Early Adolescence, 13, 4-20. 


\section{Apéndice \\ Escala de Metas Soclales en Educación Fisica (EMSEF)}

1. Intento hacer lo que el/la profesor/a me pide que haga. (RES)

2. Me gustaria llegar a conocer muy bien a mis amigos/as de clase. (REL)

3. Es importante para mi seguir las reglas de clase. (RES)

4. Me gustaria mantener las promesas que he hecho a otros/as compañeros/ as en mi clase. (REL)

5. Es importante para mi seguir trabajando incluso cuando otros/as compañeros/as no están haciendo nada. (RES)

6. Me gustaría tener un/a amigo/a en el que poder confiar. (REL)

7. Me gustaria que el/la profesor/a pensara que soy un/a estudiante responsable. (RES)

8. Me gustaría llevarme bien con la mayoría de los/as otros/as estudiantes. (REL)

9. No me gusta distraer a un/a compañero/a de clase cuando está realizando una actividad individual. (RES)

10. Es importante para mi ser aceptado/a por otros/as estudiantes. (REL)

11. Es importante para mi tener uno/a o dos amigos/as muy íntimos/as. (REL)

Nota. Los items que evalúan la meta social de responsabilidad han sido denotados con (RES) y los que miden la meta social de relación a con (REL). El formato para todos los ítems es una escala Likert de 7 puntos, de 1 (totalmente en desacuerdo) a 7(totalmente de acuerdo). El encabezamiento de todos los ítems es «En mis clases de educación física...n 